\title{
Unilateral diffuse alveolar hemorrhage in granulomatosis with polyangiitis
}

\section{Juan Molina Collada ${ }^{1}$ (D) Gema Bonilla ${ }^{1} \cdot$ Luis Gómez-Carrera $^{2}$ - Eugenio De Miguel ${ }^{1} \cdot$ Antonio Martínez Verdasco $^{2}$. Alejandro Balsa ${ }^{1}$}

Received: 21 June 2019 /Revised: 3 July 2019 / Accepted: 9 July 2019/Published online: 23 July 2019

(C) International League of Associations for Rheumatology (ILAR) 2019

\section{Presentation}

A 72-year-old woman was admitted to the emergency department with a one-week history of cough, dyspnea, and hemoptysis. Past medical history revealed a recent onset of ANA-positive oligoarthritis and that she started on methotrexate and low-dose glucocorticoids. On general examination, she was pale and auscultation revealed right-sided coarse crackles. Blood tests showed a low hemoglobin count $(8.6 \mathrm{~g} / \mathrm{dL})$ and a raised C-reactive protein $(129 \mathrm{mg} /$
L). Urine analysis was unremarkable. Chest X-ray showed unilateral alveolar infiltrates (a), and high-resolution computed tomography revealed unilateral diffuse consolidation opacities and ground-glass pattern (b, c) (Fig. 1). Diffuse alveolar hemorrhage was suspected, so bronchoscopy and bronchoalveolar lavage were undertaken. Samples came out to be hemorrhagic and positive for hemosiderin macrophages, leading the diagnosis of diffuse alveolar hemorrhage. Further analysis to rule out any systemic vasculitis showed strongly positive anti-proteinase- 3 antibodies

\section{a}

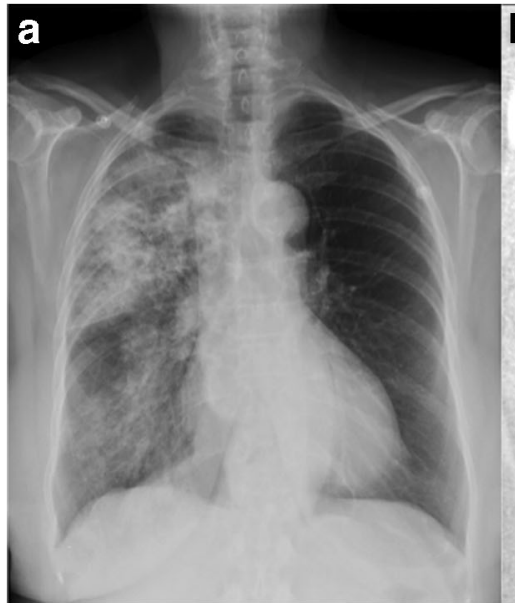

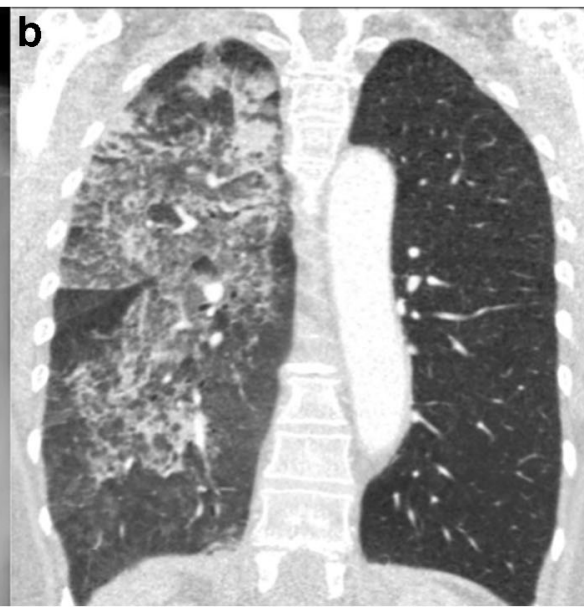

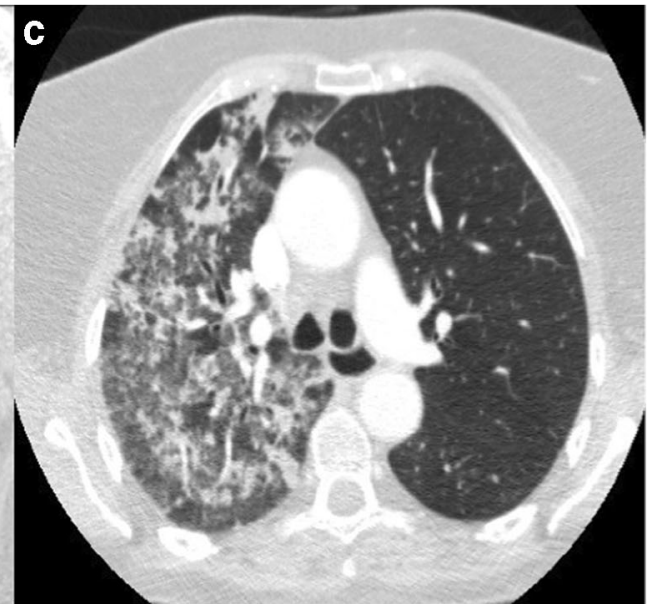

Fig. 1 Alveolar infiltrates on chest X-ray (a) and ground-glass opacities on high-resolution computed tomography (b, c) sparing the left hemithorax due to a complete unilateral right-sided diffuse alveolar hemorrhage in granulomatosis with polyangiitis

Juan Molina Collada

molinacolladajuan@gmail.com

1 Department of Rheumatology, Hospital Universitario La Paz, Madrid, Spain

2 Department of Pneumology, Hospital Universitario La Paz, Madrid, Spain 
( $85.1 \mathrm{UI} / \mathrm{mL}$, normal range $<2.0 \mathrm{IU} / \mathrm{ml}$ ) so the diagnosis of granulomatosis with polyangiitis was confirmed.

\section{Discussion}

Diffuse alveolar hemorrhage is a rare and potentially fatal manifestation of several disorders, including ANCAassociated vasculitis. The clinical presentation of diffuse alveolar hemorrhage is nonspecific, ranging from acute respiratory failure to a more insidious course, and occurs as a consequence of pulmonary capillaritis. Hemoptysis is present in two-thirds of patients, and cough, dyspnea, and fever are other frequent symptoms. Hemoglobin drop is common, and more than $50 \%$ of patients require mechanical ventilation [1]. Although mortality varies between different series, with reported survival ranging from 50 to $82 \%$ at one year $[2,3]$, diffuse alveolar hemorrhage is the strongest predictor of early mortality in ANCA-associated vasculitis. Thus, prompt diagnosis and early treatment are always required. The initial imaging evaluation of patients suspected to have diffused alveolar hemorrhage should be a plain chest X-ray; although nonspecific, it is a high sensitive investigation and $94 \%$ of patients have radiographic evidence of diffuse alveolar hemorrhage on presentation [4]. The typical radiological pattern of acute diffuse alveolar hemorrhage is characterized by consolidation or ground-glass opacities due to alveolar filling, predominantly distributed in the perihilar regions and sparing of the apices and costophrenic angles. These findings are usually diffuse and bilateral, although they may rarely be unilateral, mainly in the context of pulmonary congestion due to heart failure. High-resolution computed tomography is superior in detecting ground-glass opacities and is always required in cases of suspected diffuse alveolar hemorrhage with normal chest Xray findings. On high-resolution computed tomography, these abnormalities correspond to patchy ground-glass opacities without significant interlobular septal thickening. Within
$48 \mathrm{~h}$, septal thickening may occur and ground-glass opacities persist, resulting in a crazy-paving pattern. Pulmonary fibrosis may develop when hemorrhage recurs [5]. The highresolution computed tomography findings, although not entirely specific, can guide further investigations such as bronchoscopy and biopsy. To our knowledge, this is the first description of a complete unilateral diffuse alveolar hemorrhage due to granulomatosis with polyangiitis.

\section{Compliance with ethical standards}

Disclosures None.

Ethical approval Informed consent for publication of the case and related images was obtained from the patient.

\section{References}

1. Lichtenberger JP 3rd, Digumarthy SR, Abbott GF, Shepard J-AO, Sharma A (2014) Diffuse pulmonary hemorrhage: clues to the diagnosis. Curr Probl Diagn Radiol 43(3):128-139. https://doi.org/10. 1067/j.cpradiol.2014.01.002

2. Gallagher H, Kwan JTC, Jayne DRW (2002) Pulmonary renal syndrome: a 4-year, single-center experience. Am J Kidney Dis 39(1): 42-47. https://doi.org/10.1053/ajkd.2002.29876

3. Lauque D, Cadranel J, Lazor R, Pourrat J, Ronco P, Guillevin L, Cordier JF (2000) Microscopic polyangiitis with alveolar hemorrhage. A study of 29 cases and review of the literature. Groupe d'Etudes et de Recherche sur les maladies "Orphelines" Pulmonaires (GERM“O”P). Medicine (Baltimore) 79(4):222-233. https://doi.org/10.1097/00005792-200007000-00003

4. West S, Arulkumaran N, Ind PW, Pusey CD (2013) Diffuse alveolar haemorrhage in ANCA-associated vasculitis. Intern Med 52(1):513. https://doi.org/10.2169/internalmedicine.52.8863

5. Cortese G, Nicali R, Placido R, Gariazzo G, Anro P (2008) Radiological aspects of diffuse alveolar haemorrhage. Radiol Med 113(1):16-28. https://doi.org/10.1007/s11547-008-0229-x

Publisher's note Springer Nature remains neutral with regard to jurisdictional claims in published maps and institutional affiliations. 